\title{
Relationship between the more-affected upper limb function and daily activity performance in children with cerebral palsy: a cross-sectional study
}

\author{
Hyerin Park ${ }^{1}$, Ja Young Choi ${ }^{2}$, Sook-hee Yi ${ }^{3}$, Eun Sook Park ${ }^{1}$, Dain Shim ${ }^{1}$, Tae Young Choi ${ }^{1}$ and Dong-wook Rha ${ }^{1 *}$
}

\begin{abstract}
Background: There are differences in roles between the more-affected and less-affected upper limb of children with cerebral palsy (CP). However, there is a lack of studies of the relationship between the more-affected limb function and activities of daily living (ADL) in children with CP. Thus, the aim of this prospective cross-sectional study was to investigate the relationship between more-affected upper limb function and ADL in children with CP.

Methods: Children with spastic CP (unilateral $C P n=28$, bilateral $C P n=31 ; 34$ males, 25 females; mean age $\pm S D$, $6.8 \pm 3.1 y$ [range, 3-14y]) participated in this study. Function of the more-affected upper limb was measured using the Melbourne Assessment of Unilateral Upper limb Function, version 2 (MA2) and the Upper Limb Physician's Rating Scale (ULPRS). Performance of daily living activities was measured using the Pediatric Evaluation of Disability Inventory-Computer Adaptive Test (PEDI-CAT).

Results: The range, accuracy and fluency dimension of MA2 and ULPRS total scores were moderately correlated with the daily activity domain ( $r=0.47,0.47,0.56$ for MA2 and $r=0.50$ for ULPRS, respectively; $P<0.001)$ rather than the mobility, social/cognitive, and responsibility domains of the PEDI-CAT. ULPRS scores for elbow extension, supination in extension, supination in flexion, and two-handed function were moderately correlated with the PEDI-CAT daily activity domain $(r=0.44,0.43,0.41$, and 0.49 , respectively; $P<0.01)$. Finger opening and thumb-in-palm deformity of the ULPRS did not correlate with any PEDI-CAT domain.

Conclusions: The MA2 range, accuracy, and fluency domains (rather than dexterity) had the strongest correlations with the PEDI-CAT daily activity domain. Elbow extension, forearm supination, and two-handed function (rather than wrist and finger movements) of the ULPRS had the strongest correlations with the PEDI-CAT daily activity domain.
\end{abstract}

Keywords: Cerebral palsy, Activities of daily living, Pediatric rehabilitation

*Correspondence: medicus@yuhs.ac

${ }^{1}$ Department and Research Institute of Rehabilitation Medicine, Yonsei University College of Medicine, 50-1, Yonsei-ro, Seodaemun-gu, Seoul, Republic of Korea

Full list of author information is available at the end of the article

\section{Background}

Children with cerebral palsy (CP) commonly have impairment of upper limb function, with various studies reporting a prevalence ranging from 57 to $83 \%[1,2]$. Children with spastic CP can be classified into unilateral or bilateral distribution, and both groups frequently display upper limb function impairment [2]. 
There is often an asymmetry in upper limb function in children with bilateral or unilateral $\mathrm{CP}$, with one limb more severely affected than the other $[3,4]$. Most activities of daily living (ADL) are bimanual, but the division of labor may be different for the more-affected and less-affected limbs. In typically developing children, the dominant hand is used for precision tasks, and the non-dominant hand is used for stabilizing tasks [5]. For example, when writing, a child will grip a pencil with the dominant hand and hold the paper with the other. Similarly, as children with $\mathrm{CP}$ prefer to use their less-affected limb for ADL, we expect to observe differences in the functional roles between the more-affected and lessaffected limbs; the more-affected limb used as the nondominant hand, and the less-affected limb used as the dominant hand.

A number of interventions (e.g., neuromuscular electrical stimulation, botulinum toxin injections, occupational therapy, orthoses) for the upper limb have been reported in children with unilateral and bilateral CP $[6,7]$. The aims of interventions targeting upper limb function in children with $\mathrm{CP}$ are to improve functional abilities and increase independence in ADL [6]. The ADL refer to the ability to perform self-care and daily activities, corresponding to activity and participation components of the International Classification of Functioning, Disability, and Health (ICF) [8]. The ICF provides a framework for measuring dysfunction according to level of impairment (structural), activity limitations (difficulty in task execution), and participation restrictions (degree of activity involvement).

Little is known about the relationship between the function of the more-affected limb and ADL in children with $\mathrm{CP}$, although previous studies report a significant relationship between self-care activities and overall manual ability [9-12]. Impaired upper limb function can restrict ADL participation in young adults with CP [13], but this study only analyzed the relationship between $\mathrm{ADL}$ and overall upper limb function measured by the Manual Ability Classification System (MACS) and did not evaluate the function of the more-affected upper limb. One report examined the relationship between affected limb function and ADL in unilateral CP, although the approach to ADL classification was simplistic, the participation-restriction category was not examined [12], and no information was provided for how the movement patterns of the affected limbs influenced the performance of ADL.

This study addressed two questions. First, are there any relationships between the four Pediatric Evaluation of Disability Inventory-Computer Adaptive Test (PEDICAT) domains (daily activities, mobility, social/cognitive, responsibility) and more-affected limb function in children with spastic CP? Second, if these relationships are identified, which movement patterns of the moreaffected limb are associated with ADL performance?

\section{Methods \\ Participants}

This cross-sectional study was conducted at three pediatric rehabilitation institutions in the Republic of Korea from July 2017 to March 2018. The study included 59 children with spastic $\mathrm{CP}$ with upper limb dysfunction who met the inclusion and exclusion criteria: unilateral CP, $n=28$; bilateral CP, $n=31$; 34 males and 25 females; mean age 6 years 8 months, SD 3 years 1 month). The two inclusion criteria were as follows: (1) actively use their arm with manual ability classification system (MACS) levels I to IV and House Functional Classification (HFC) levels 4 to 7, (2) age 3-18years. The three exclusion criteria were as follows: (1) children with severe mental retardation who could not understand instructions; (2) history of visual impairment; (3) history of operations and/or botulinum toxin injections in an upper limb, or received constraint-induced movement therapy (CIMT) within 6 months of beginning the study. This study was approved by the Institutional Review Boards at all participating hospitals (Severance Hospital, Eulji University Hospital, and Seoul Rehabilitation Hospital). All participants' parents provided written informed consent. The trial was registered at the Clinical Research Information Service (identifier no. КСТ0002395).

\section{Procedure}

One occupational therapist (an author of this study, DS) performed all assessments to exclude inter-rater bias. The Melbourne Assessment of Unilateral Upper Limb Function version 2 (MA2) and the Upper Limb Physician's Rating Scale (ULPRS) were used to measure limb functions in the more-affected/non-dominant upper limb. The performance of ADL was measured using PEDICAT. The MA2 percent score and the PEDI-CAT scaled score were then used for the analyses. In cases of children with bilateral $\mathrm{CP}$, we used data from the non-dominant limb.

\section{Melbourne assessment of unilateral upper limb function version 2}

The MA2 is a well-known tool for measuring unilateral upper limb function in children with neurological impairments aged 2.5-15years old [14]. The MA2 has 30 possible scores in four dimensions based on 14 tasks of reaching, grasping, releasing, and manipulating objects: (1) range of motion (ROM), (2) accuracy, (3) dexterity, and (4) fluency of upper limb function. MA2 has high inter-rater, intra-rater, and test-retest reliability 
[intraclass correlation coefficient $(\mathrm{ICC})=0.90-1.00]$ for the four subscale scores [15], and video is taken during testing for accurate scoring [16]. The scoring system uses a 3-, 4-, or 5-point scale, with lower scores representing worse impairment. A raw score is the sum of each individual score, and the percent score represents the raw score divided by the maximum total score, multiplied by 100 [17]. We computed the percent score for these analyses.

\section{Upper limb Physician's rating scale assessment}

The ULPRS is a semiquantitative assessment that evaluates movement patterns and determines functional impairment of the upper limb by focusing on all three arm levels, including the palm, forearm, and elbow (Supplemental Table S1) [18]. The advantages of the ULPRS include its ease of use by healthcare providers without the need for special training, and high inter-rater and intra-rater reliability $(\mathrm{ICC}=0.94$ between raters, and ICC $=0.99-1.00$ within raters) [19]. Among other parameters, the ULPRS assessment can determine whether there is an isolated functional impairment, such as restricted forearm supination, a wrist in flexion and deviation, elbow flexion, or thumb-in-palm, with unilateral limb scores ranging from 0 to 25 (lower scores represent worse impairment).

\section{Pediatric evaluation of disability inventory-computer adaptive test}

The PEDI-CAT is a standardized outcome measure that assesses functional activities in children and young people $[20,21]$. The PEDI-CAT is a revised version of the original Pediatric Evaluation of Disability Inventory (PEDI). The original PEDI was used as a comprehensive functional assessment of key activity performance in children aged 6 months to 7.5 years, whereas the PEDI-CAT is recommended for assessing children aged 0 to 21 years with different health conditions. The PEDI-CAT incorporates a computer-adaptive platform with 276 items based on parental or caregiver reporting, and has four domains that cover daily activities, mobility, social/cognitive function, and responsibility [22].

The PEDI-CAT was designed to be consistent with the ICF and corresponds to the activities and participation components of the ICF [23]. The daily activity, mobility, and social/cognitive domains of the PEDI-CAT all correspond to activity dimensions in the ICF (defined by the performance of a variety of tasks), and the PEDI-CAT responsibility domain corresponds to the ICF participation dimension (defined as being involved in real-life situations) [22].

The PEDI-CAT outcome measure has strong reliability and construct validity in children with CP [24]. There are two versions of the assessment: a "speedy" PEDICAT, and a content-balanced PEDI-CAT. We used the speedy version because of its efficiency. A scaled score for each domain (ranging from 20 to 80 ) was used for the analyses (lower scaled scores represent lower functional activities).

\section{Statistical analysis}

Data were analyzed using the Statistical Package for the Social Sciences Program (SPSS version 23.0). The Shapiro-Wilk test was used to test for a normal distribution of the data. Pearson and Spearman correlation coefficients were calculated to estimate correlations between the MA2 scores and each of the four PEDI-CAT domain scaled scores. Spearman correlation coefficients were used to calculate correlations between ULPRS scores and each of the four PEDI-CAT domain scaled scores. We calculated partial correlation coefficients to control for the potential confounders of age and Gross Motor Function Classification System (GMFCS) levels. Correlation coefficient values of $<0.2,0.2-0.4,0.4-0.6,0.6-0.8$, and $>0.8$ were considered as very weak, weak, moderate, strong, and very strong, respectively [25]. We considered correlation coefficient values $>0.4$ as meaningful.

\section{Results \\ Participant characteristics}

A total of 59 children with spastic CP (34 males and 25 females) aged 3 to 14 years (mean age 6 years 8 months, SD 3 years 1 month) were included in this study. Twentyeight children had unilateral $\mathrm{CP}$, and 31 children had bilateral CP. The descriptive characteristics of the participants including age, sex, more-affected side, involved side, and distribution of GMFCS, MACS, and HFC levels are presented in Table 1.

Table 1 Participant characteristics

\begin{tabular}{ll}
\hline Characteristic & Number/Value $^{\mathbf{a}^{2}}$ \\
\hline Number of participants & 59 \\
Age at assessment & $6.8 \pm 3.1(3-14)$ \\
More-affected side, right/left & $31 / 28$ \\
Sex, male/female & $34 / 25$ \\
Involved side, unilateral/bilateral & $28 / 31$ \\
GMFCS level, I/II/III/IV & $25 / 19 / 8 / 7$ \\
MACS level, I/II/II/IV & $2 / 25 / 18 / 14$ \\
HFC 4/5/6/7 & $14 / 15 / 22 / 8$ \\
\hline
\end{tabular}

GMFCS, Gross Motor Function Classification System; MACS, Manual Ability Classification System; HFC, House Functional Classification

a Values are mean $\pm \mathrm{SD}$ (range) 


\section{MA2 and PEDI-CAT correlations}

Partial correlations between MA2 scores and PEDI-CAT domains are presented in Table 2. Scatter diagrams of the PEDI-CAT daily activity domain plotted against MA2 range, accuracy, dexterity, and fluency dimension scores are presented in Fig. 1.

MA2 range, accuracy, and fluency dimensions were moderately positively correlated with the PEDI-CAT daily activity domain $(\mathrm{r}=0.47,0.47,0.56$, respectively; $P<0.001$ ), and were weakly to moderately correlated with the PEDI-CAT responsibility domain $(\mathrm{r}=0.32,0.30,0.40$, respectively; $P<0.05)$. The MA2 dexterity dimension was weakly correlated with the PEDI-CAT daily activity domain, whereas it was not significantly correlated with the PEDI-CAT mobility, social/cognitive, and responsibility domains. No MA2 dimension correlated with the PEDI-CAT social/cognitive domain.

\section{ULPRS and PEDI-CAT correlations}

Partial correlations between ULPRS scores and PEDICAT domains are presented in Table 3. Active elbow extension, active supination in elbow extension, and active supination in flexion were moderately positively correlated with the PEDI-CAT daily activity domain

Table 2 Partial correlation coefficients between the MA2 and the PEDI-CAT

\begin{tabular}{|c|c|c|c|c|}
\hline & \multicolumn{4}{|l|}{ PEDI-CAT } \\
\hline & $\begin{array}{l}\text { Daily } \\
\text { activity }\end{array}$ & Mobility & $\begin{array}{l}\text { Social/ } \\
\text { cognitive }\end{array}$ & Responsibility \\
\hline \multicolumn{5}{|l|}{ MA2 } \\
\hline \multicolumn{5}{|l|}{ Range } \\
\hline Correlation & $0.47^{\mathrm{a}}$ & 0.26 & 0.19 & 0.32 \\
\hline$P$-value & $<0.001^{* *}$ & 0.056 & 0.167 & $0.014^{*}$ \\
\hline \multicolumn{5}{|l|}{ Accuracy } \\
\hline Correlation & $0.47^{\mathrm{a}}$ & 0.22 & 0.24 & 0.30 \\
\hline$P$-value & $<0.001^{* *}$ & 0.095 & 0.072 & $0.022^{*}$ \\
\hline \multicolumn{5}{|l|}{ Dexterity } \\
\hline Correlation & 0.38 & 0.13 & 0.12 & 0.12 \\
\hline$P$-value & $0.004^{* *}$ & 0.355 & 0.391 & 0.390 \\
\hline \multicolumn{5}{|l|}{ Fluency } \\
\hline Correlation & $0.56^{\mathrm{a}}$ & $0.40^{\mathrm{a}}$ & 0.23 & $0.40^{\mathrm{a}}$ \\
\hline$P$-value & $<0.001^{* *}$ & $0.002^{* *}$ & 0.083 & $0.002^{* *}$ \\
\hline
\end{tabular}

MA2, Melbourne Assessment of Unilateral Upper Limb Function, version 2; PEDICAT, Pediatric Evaluation of Disability Inventory-Computer Adaptive Test

Partial correlation coefficients are adjusted by age and GMFCS levels and are

Spearman's $r$ values for daily activity, mobility and Pearson's $r$ values for social/ cognitive, and responsibility domains of the PEDI-CAT

${ }^{*}$ Two-tailed significance, $P<0.05$

** Two-tailed significance, $P<0.01$

Significant $P$-values are highlighted in bold font

a Correlation coefficient value $0.4-0.6$; moderate correlation
( $\mathrm{r}=0.44,0.43$, and 0.41 , respectively; $P<0.01$ ). The twohanded function score had the highest correlation coefficient for the PEDI-CAT daily activity domain $(r=0.49$; $P<0.001)$. Finger opening and thumb-in-palm were not significantly correlated with the PEDI-CAT daily activity domain. The PEDI-CAT responsibility domain was only weakly correlated with each ULPRS domain, except for finger and thumb movement.

\section{Discussion}

The first aim of this study was to examine the relationships between the four PEDI-CAT domains and the functionality of the more-affected limb in children with spastic CP. We identified moderately positive correlations between the PEDI-CAT daily activity domain and the function of the more-affected upper limb in children with CP. There was no significant correlation between the PEDI-CAT social/cognitive domain and the function of the more-affected upper limb in children with CP.

The results indicated that upper limb function in children with CP (assessed using MA2 and ULPRS) was primarily correlated with the PEDI-CAT daily activity domain rather than other domains. Real-life hand skill performance was reported to be a contributing factor of self-care function in children with and without disabilities [26]. Previous research reported a strong correlation between upper-extremity function (assessed using the original Melbourne Assessment) and the PEDI self-care domain [17], which is consistent with our results correlating the PEDI-CAT daily activity domain (equivalent to the original PEDI functional skills of self-care domain) with upper-extremity function. However, the previous study had smaller sample size, did not analyze other ADL domains, and did not specifically target the function of the more-affected limb [17]. Our results showed the importance of improving the function of the moreaffected upper limb to enhance daily activities, such as dressing, eating, and grooming. The PEDI-CAT responsibility domain was only weakly correlated with MA2 values for children with CP. MA2 is a tool that measures unimanual capacities, and the PEDI-CAT responsibility domain corresponds with the ICF participation dimension. Therefore, a weak association between MA2 and PEDI-CAT responsibility domain might indicate a gap between participant unimanual capacities and their participation.

We found no significant relationship between upper limb function in children with $\mathrm{CP}$ and the PEDI-CAT social/cognitive domain. This domain represents the ability to interact with people, and includes the skills needed for effective social exchanges and to function safely [22]. Social participation impairments in children with CP are related to intellectual function, environmental factors, 

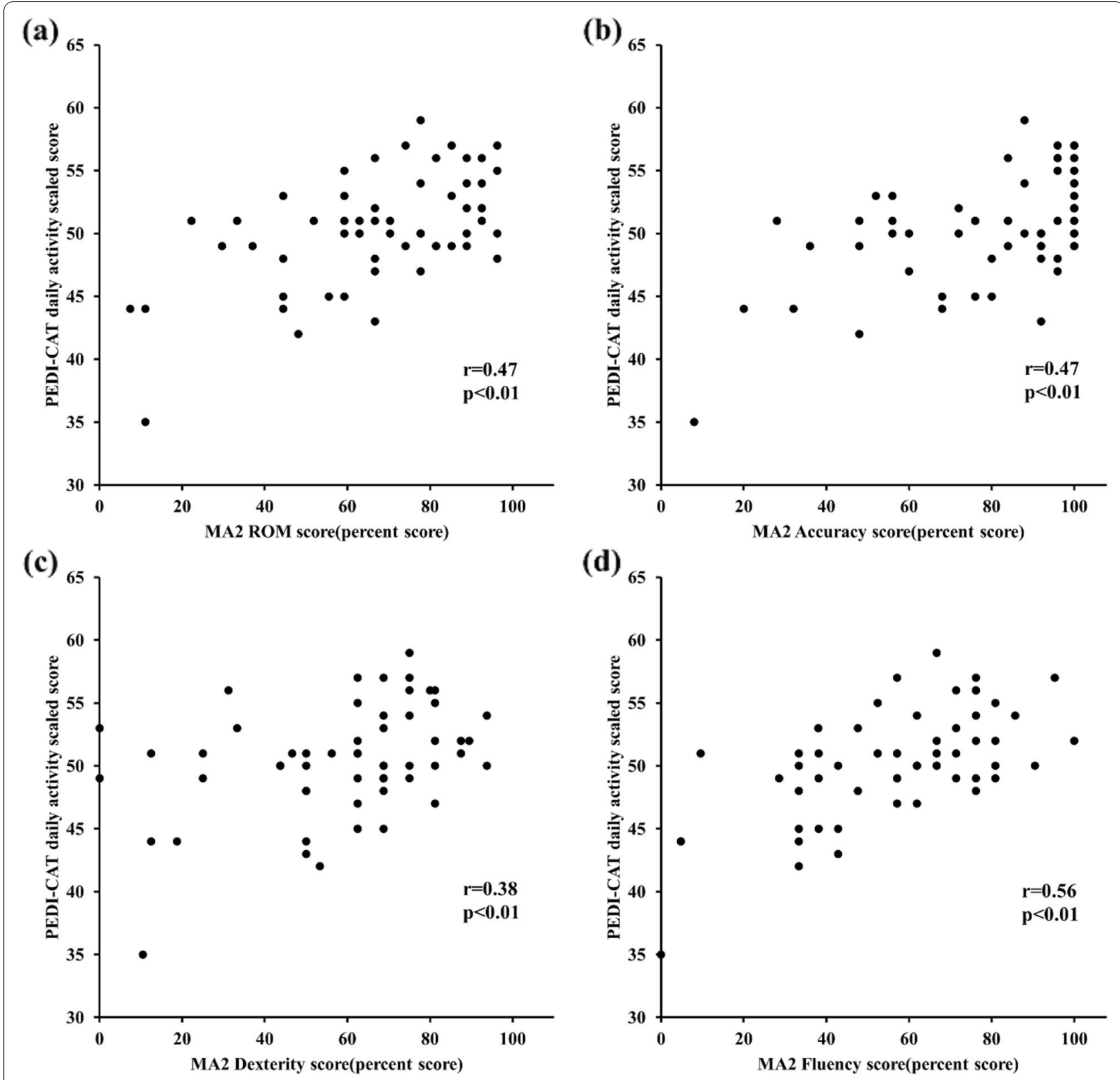

Fig. 1 Correlation between MA2 dimensions and PEDI-CAT daily activity score. a MA2 ROM percent score correlation with PEDI-CAT daily activity scaled score $(r=0.47, P<0.01)$. b MA2 accuracy percent score correlation with PEDI-CAT daily activity scaled score $(r=0.47, P<0.01)$. $\mathbf{c}$ MA2 dexterity percent score correlation with PEDI-CAT daily activity scaled score $(r=0.38, P<0.01)$. d MA2 fluency percent score correlation with PEDI-CAT daily activity scaled score $(r=0.56, P<0.01)$

and motor impairment [27, 28]. Therefore, the PEDICAT social/cognitive domain might be affected by a variety of factors. Further studies are needed to examine the factors that affect social and cognitive aspects of behavior in children with $\mathrm{CP}$.

The second aim of this study was to identify the movement patterns of the more-affected limb that were related to the four PEDI-CAT domains in children with CP. We found that there was weak association between dexterity and PEDI-CAT daily activities, compared to moderate correlations between MA2 domains (range, accuracy, and fluency) and PEDI-CAT daily activities. The dexterity test consists of grasp/release of crayon, grasp/release of pellet, and manipulation, all of which are related to finger movement. ULPRS assessments for finger opening and thumb-in-palm were not significantly correlated 
Table 3 Partial correlation coefficients between the ULPRS and the PEDI-CAT

\begin{tabular}{|c|c|c|c|}
\hline \multicolumn{4}{|c|}{ PEDI-CAT } \\
\hline $\begin{array}{l}\text { Daily } \\
\text { activity }\end{array}$ & Mobility & $\begin{array}{l}\text { Social/ } \\
\text { cognitive }\end{array}$ & Responsibility \\
\hline
\end{tabular}

\section{ULPRS}

Active elbow extension

Correlation $0.44^{\mathrm{a}}$

P-value $\quad \mathbf{0 . 0 0 1}^{* *}$

$0.32 \quad 0.24$

$\mathbf{0 . 0 1 5 ^ { * }} \quad 0.071$

0.36

Active supination in extension

$\begin{array}{ll}\text { Correlation } & 0.43^{\mathrm{a}} \\ \text { P-value } & \mathbf{0 . 0 0 1}\end{array}$

Active supination in flexion

Correlation $0.41^{\mathrm{a}}$

$P$-value $\quad \mathbf{0 . 0 0 2}^{* *}$

0.33

$0.012^{*}$

0.24

0.070

$0.40^{\mathrm{a}}$

$0.002^{* *}$

0.23

Active wrist dorsiflexion

Correlation 0.27

$P$-value $\quad \mathbf{0 . 0 4 5 ^ { * }}$

0.13

0.336

0.23

Wrist deviation

Correlation 0.36

$P$-value $\quad \mathbf{0 . 0 0 6} 6^{* *}$

0.23

0.088

$$
0.31
$$

$0.019^{*}$

$0.004^{* *}$

Finger opening

Correlation 0.06

P-value $\quad 0.677$

$-0.03$

0.08

0.562

\subsection{5}

0.260

Thumb in palm

Correlation 0.22

$-0.04$

0.06

0.13

$P$-value $\quad 0.107$

0.796

0.669

\subsection{4}

Associated increase in muscle tone

\begin{tabular}{|c|c|c|c|c|}
\hline Correlation & 0.38 & 0.29 & 0.05 & 0.21 \\
\hline$P$-value & $0.004^{* *}$ & $0.026^{*}$ & 0.713 & 0.117 \\
\hline \multicolumn{5}{|c|}{ Wwo-handed function } \\
\hline Correlation & $0.49^{\mathrm{a}}$ & 0.38 & 0.25 & 0.35 \\
\hline $\begin{array}{c}\text { P-value } \\
\text { Total score }\end{array}$ & $<0.001^{* *}$ & $0.003^{* *}$ & 0.059 & $0.008^{* *}$ \\
\hline Correlation & $0.50^{\mathrm{a}}$ & 0.31 & 0.28 & $0.42^{\mathrm{a}}$ \\
\hline$P$-value & $<0.001^{* *}$ & $0.021^{*}$ & $0.037^{*}$ & $0.001^{* *}$ \\
\hline
\end{tabular}

ULPRS, Upper Limb Physician's Rating Scale; PEDI-CAT, Pediatric Evaluation of Disability Inventory-Computer Adaptive Test

Partial correlation coefficients are adjusted by age and GMFCS levels and are Spearman's $r$ values. Significant $P$-values are highlighted in bold font

${ }^{*}$ Two-tailed significance, $P<0.05,{ }^{* *}$ Two-tailed significance, $P<0.01$

${ }^{a}$ Correlation coefficient value $0.4-0.6$; moderate correlation

with the PEDI-CAT daily activity domain. Finger opening and thumb-in-palm are related to articular movements of the hand and fingers, which are considered as fine motor skills. Our results indicated that fine motor skills (smaller movements that occur in the hands and fingers) in the more-affected upper limb were not significantly or weakly correlated with the PEDI-CAT daily activity domain.
Most ADL consist of bimanual activities, and the roles of dominant and non-dominant hands are very different for these everyday activities. Children with $\mathrm{CP}$ use the more-affected hand to assist in bimanual activities, and use the less-affected hand for unimanual activities. Differences in function between the more and less-affected hands can lead to their different roles in daily activities.

Children with unilateral CP prefer to use the lessaffected hand for precision tasks and the more-affected hand (the assisting hand) for complementary tasks, such as holding and stabilizing [29]. A previous study reported that the more-affected hand was used to provide fixation (usually requires fewer fine motor skills), whereas the less-affected hand was used for manipulation (associated with fine motor skills) [30]. However, these earlier reports only examined assisting hand preferences, and did not provide information about the assisting hand movement patterns that affected ADL. Our present results suggest that the assisting hand movement patterns affecting ADL were more related to complementary tasks than precision tasks.

The functional ULPRS assessment of the more-affected upper limb showed that PEDI-CAT daily activities were more closely related to proximal-joint motions (elbow extension and forearm supination) than to distal-joint motions of the wrist and hand. The fine motor skills in the wrists and hands are related to precision tasks, whereas proximal-joint movements are more relevant for complementary tasks than detailed tasks. Our results showed that ADL was more closely correlated with proximal-joint movements than distal joint movements of the more-affected limb. We propose that more consideration should be given to rehabilitation programs that provide therapy for the more-affected limb to act as an assisting hand for performing complementary tasks rather than performing precision tasks, which could improve ADL in children with CP.

Klingels et al. [31] described the association between distal upper limb impairments (distal muscle strength and grip strength) and activity measures. Mailleux et al. [32] reported the importance of distal limb movements for the execution of functional tasks. These discrepancies might be explained by the wide age ranges and severity of upper limb function impairment in the studies. Further studies are needed to verify these results, such as a comparative analysis of the effects of botulinum toxin injections on elbow flexors and pronators versus injections into wrist flexors and thumb adductors.

Another important finding was that ULPRS twohanded function scores were moderately correlated with the PEDI-CAT daily activity domain. Bimanual performance is significantly associated with ADL motor skills in unilateral CP [12], and a strong positive association 
between self-care and bimanual performance was reported [33]. Previous CIMT and Hand Arm Bimanual Intensive Training (HABIT) studies also revealed the importance of bimanual training for improving $\mathrm{ADL}$ in children with CP $[34,35]$. CIMT and HABIT are frequently used as interventions for upper limb training in children with CP. CIMT restrains the non-affected limb to promote the use of the affected limb, and HABIT is an intensive treatment that provides bimanual activities to improve bimanual coordination. CIMT has achieved greater efficacy than HABIT in improving impaired arm function in children with hemiplegic $\mathrm{CP}$, although HABIT achieved greater improvement in bimanual performance and ADL than CIMT [34, 35]. Considering our results and those of previous studies, it might be helpful to implement bimanual and unimanual training to improve ADL, and two-handed function should be assessed before and after any intervention for the moreaffected limb.

A limitation of this study was that the more-affected limb function was primarily assessed during unimanual activities, so further studies are needed to use existing tools to evaluate the function of the affected limb during bimanual activities. For example, the Assisting Hand Assessment (AHA) can be performed to measure the effectiveness of the assisting hand in bimanual activities of children with unilateral impairment [29]. The Both Hands Assessment (BoHA) can be used to measure the effectiveness of bimanual performance and the extent of asymmetric hand use in children with bilateral cerebral palsy [36]. The correlations between upper limb function and the PEDI-CAT daily activity domain were not strong; therefore, follow-up studies on other factors affecting ADL are needed. As this study was a cross-sectional design, the direction of causality between the function of the more-affected upper limb and daily activity performance remains unclear, and the results may not be generalizable. Nevertheless, we believe our results provide useful insights into upper limb interventions to improve ADL. Further studies to investigate whether rehabilitation management for upper limb training for the assisting hand during bimanual activities can enhance ADL, which may help to clarify any relationships between the affected upper limb function and the performance of ADL in children with $\mathrm{CP}$.

\section{Conclusions}

This study demonstrated that the functionality of the more-affected upper limb (assessed by MA2 and ULPRS) correlated with the performance of ADL in children with spastic CP. MA2 and ULPRS assessments were significantly correlated with the PEDI-CAT daily activity domain, but not with the PEDI-CAT social/ cognitive domain. The MA2 range, accuracy, and fluency dimensions (rather than dexterity), and the elbow extension, forearm supination, and two-handed function (rather than wrist and finger movements) assessed by the ULPRS displayed the strongest correlations with the PEDI-CAT daily activity domain.

\begin{abstract}
Abbreviations
CP: cerebral palsy; MA2: Melbourne Assessment of Unilateral Upper Limb Function version 2; GMFCS: Gross Motor Function Classification System; MACS, Manual Ability Classification System; PEDI-CAT: Pediatric Evaluation of Disability Inventory-Computer Adaptive Test; ULPRS: Upper Limb Physician's Rating Scale; CIMT: Constraint-Induced Movement Therapy; HABIT: Hand Arm Bimanual Intensive Training; AHA: Assisting Hand Assessment; BoHA: Both Hands Assessment.
\end{abstract}

\section{Supplementary Information}

The online version contains supplementary material available at https://doi. org/10.1186/s12887-021-02927-2.

Additional file 1: Supplemental Table S1. Upper Limb Physician's Rating Scale. Upper Limb Physician's Rating Scale.

Acknowledgements

Not applicable.

Authors' contributions

HP, JYC, SY, ESP, DS, TYC, and DR participated in study design and data analysis and interpretation. JYC was involved in the original draft preparation. HP was involved in writing the manuscript. HP, JYC, SY, ESP, and DR were involved in reviewing and editing the final manuscript. All authors read and approved the final manuscript.

\section{Funding}

This study was supported by the Korea Health Technology R\&D Project through the Korea Health Industry Development Institute (KHIDI) and funded by the Ministry of Health and Welfare, Republic of Korea (grant number: HI17C0391)

\section{Availability of data and materials}

The data sets generated and analyzed during the current study are not publicly available due to the privacy of research participants, but are available from the corresponding author on reasonable request.

\section{Declarations}

\section{Ethics approval and consent to participate}

This study was approved by the Institutional Review Boards at all participating hospitals. All participants' parents provided written informed consent. The IRB numbers of the participating hospitals are as follows: 1-2015-0097 at Yonsei University, Health System, Severance Hospital, Seoul, Republic of Korea; EMC2017-05-002 at Eulji University Hospital, Daejeon, Republic of Korea; and SRH2017R-02 at Seoul Rehabilitation Hospital, Seoul, Republic of Korea. The study was conducted in accordance with The Code of Ethics of the World Medical Association (Declaration of the Helsinki) for the involvement of humans.

Consent for publication

Not applicable.

Competing interests

The authors declare that they have no competing interests. 


\section{Author details}

'Department and Research Institute of Rehabilitation Medicine, Yonsei University College of Medicine, 50-1, Yonsei-ro, Seodaemun-gu, Seoul, Republic of Korea. ${ }^{2}$ Department of Rehabilitation Medicine, Chungnam National University College of Medicine, 282, Munhwa-ro, Jung-gu, Daejeon, Republic of Korea. ${ }^{3}$ Department of Rehabilitation Medicine, Seoul Rehabilitation Hospital, 30, Galhyeon-ro 11-gil, Eunpyeong-gu, Seoul, Republic of Korea.

Received: 11 December 2020 Accepted: 22 September 2021 Published online: 19 October 2021

\section{References}

1. Makki D, Duodu J, Nixon M. Prevalence and pattern of upper limb involvement in cerebral palsy. J Child Orthop. 2014;8(3):215-9.

2. Arner M, Eliasson AC, Nicklasson S, Sommerstein K, Hägglund G. Hand function in cerebral palsy. Report of 367 children in a population-based longitudinal health care program. J Hand Surg Am. 2008;33(8):1337-47.

3. Basu AP, Pearse JE, Baggaley J, Watson RM, Rapley T. Participatory design in the development of an early therapy intervention for perinatal stroke. BMC Pediatr. 2017;17(1):33.

4. Rich TL, Menk JS, Rudser KD, Feyma T, Gillick BT. Less-affected hand function in children with Hemiparetic unilateral cerebral palsy: a comparison study with typically developing peers. Neurorehabil Neural Repair. 2017;31(10-11):965-76.

5. Fagard J, Jacquet A-Y. Onset of bimanual coordination and symmetry versus asymmetry of movement. Infant Behav Dev. 1989;12(2):229-35.

6. Plasschaert VFP, Vriezekolk JE, Aarts PBM, Geurts ACH, Van den Ende CHM. Interventions to improve upper limb function for children with bilateral cerebral palsy: a systematic review. Dev Med Child Neurol. 2019;61(8):899-907

7. Sakzewski L, Ziviani J, Boyd RN. Efficacy of upper limb therapies for unilateral cerebral palsy: a meta-analysis. Pediatrics. 2014;133(1):e175-204.

8. Organization WH. IFC: international classification of functioning, disability and health. 2001.

9. Kuijper MA, van der Wilden GJ, Ketelaar M, Gorter JW. Manual ability classification system for children with cerebral palsy in a school setting and its relationship to home self-care activities. Am J Occup Ther. 2010;64(4):614-20.

10. Gunel MK, Mutlu A, Tarsuslu T, Livanelioglu A. Relationship among the manual ability classification system (MACS), the gross motor function classification system (GMFCS), and the functional status (WeeFIM) in children with spastic cerebral palsy. Eur J Pediatr. 2009:168(4):477-85.

11. Öhrvall AM, Eliasson AC, Löwing K, Ödman P, Krumlinde-Sundholm L. Self-care and mobility skills in children with cerebral palsy, related to their manual ability and gross motor function classifications. Dev Med Child Neurol. 2010;52(11):1048-55.

12. James S, Ziviani J, Ware RS, Boyd RN. Relationships between activities of daily living, upper limb function, and visual perception in children and adolescents with unilateral cerebral palsy. Dev Med Child Neurol. 2015;57(9):852-7.

13. van Meeteren J, Roebroeck ME, Celen E, Donkervoort M, Stam HJ. Functional activities of the upper extremity of young adults with cerebral palsy: a limiting factor for participation? Disabil Rehabil. 2008;30(5):387-95.

14. Randall M, Imms C, Carey LM, Pallant JF. Rasch analysis of the Melbourne assessment of unilateral upper limb function. Dev Med Child Neurol. 2014;56(7):665-72.

15. Gerber CN, Plebani A, Labruyère R. Translation, reliability, and clinical utility of the Melbourne assessment 2. Disabil Rehabil. 2019;41(2):226-34

16. Randall M, Johnson L, Reddihough D. The Melbourne Assessment 2 : About the Melbourne Assessment 2. https://www.rch.org.au/melbournea ssessment. Accessed 06282020

17. Bourke-Taylor H. Melbourne assessment of unilateral upper limb function: construct validity and correlation with the pediatric evaluation of disability inventory. Dev Med Child Neurol. 2003;45(2):92-6.

18. Graham HK, Aoki KR, Autti-Rämö I, Boyd RN, Delgado MR, Gaebler-Spira $D J$, et al. Recommendations for the use of botulinum toxin type a in the management of cerebral palsy. Gait Posture. 2000;11(1):67-79.
19. Park ES, Joo JW, Kim SA, Rha DW, Jung SJ. Reliability and validity of the upper limb Physician's rating scale in children with cerebral palsy. Yonsei Med J. 2015;56(1):271-6.

20. Dumas HM, Fragala-Pinkham MA, Haley SM. Development of a postacute hospital item bank for the new pediatric evaluation of disability inventory-computer adaptive test. Int J Rehabil Res. 2010;33(4):332-8.

21. Haley SM, Coster WJ, Dumas HM, Fragala-Pinkham MA, Kramer J, Ni P, et al. Accuracy and precision of the pediatric evaluation of disability inventory computer-adaptive tests (PEDI-CAT). Dev Med Child Neurol. 2011;53(12):1100-6.

22. Haley SM, Coster WJ, Dumas HM, Fragala-Pinkham MA, Moed R. PEDI-CAT (version 1.4.0): development, standardization and administration manual. Boston: Trustees of Boston University; 2015.

23. Thompson SV, Cech DJ, Cahill SM, Krzak JJ. Linking the pediatric evaluation of disability inventory-computer adaptive test (PEDICAT) to the international classification of function. Pediatr Phys Ther. 2018;30(2):113-8

24. Shore BJ, Allar BG, Miller PE, Matheney TH, Snyder BD, Fragala-Pinkham M. Measuring the reliability and construct validity of the pediatric evaluation of disability inventory-computer adaptive test (PEDI-CAT) in children with cerebral palsy. Arch Phys Med Rehabil. 2019;100(1):45-51.

25. Franzblau AN. A primer of statistics for non-statisticians. Harcourt, Brace: Oxford, England; 1958.

26. Chien CW, Brown T, McDonald R, Yu ML. The contributing role of real-life hand skill performance in self-care function of children with and without disabilities. Child Care Health Dev. 2014;40(1):134-44

27. Mihaylov SI, Jarvis SN, Colver AF, Beresford B. Identification and description of environmental factors that influence participation of children with cerebral palsy. Dev Med Child Neurol. 2004;46(5):299-304.

28. Bottcher L. Children with spastic cerebral palsy, their cognitive functioning, and social participation: a review. Child Neuropsychol. 2010;16(3):209-28.

29. Krumlinde-Sundholm L, Eliasson A-C. Development of the assisting hand assessment: a Rasch-built measure intended for children with unilateral upper limb impairments. Scand J Occup Ther. 2003;10(1):16-26.

30. Lemmens RJ, Janssen-Potten YJ, Timmermans AA, Defesche A, Smeets RJ, Seelen HA. Arm hand skilled performance in cerebral palsy: activity preferences and their movement components. BMC Neurol. 2014;14:52.

31. Klingels K, Demeyere I, Jaspers E, De Cock P, Molenaers G, Boyd R, et al. Upper limb impairments and their impact on activity measures in children with unilateral cerebral palsy. Eur J Paediatr Neurol. 2012;16(5):475-84.

32. Mailleux L, Jaspers E, Ortibus E, Simon-Martinez C, Desloovere K, Molenaers $G$, et al. Clinical assessment and three-dimensional movement analysis: an integrated approach for upper limb evaluation in children with unilateral cerebral palsy. PLoS One. 2017;12(7):e0180196.

33. Burgess A, Boyd RN, Chatfield MD, Ziviani J, Wotherspoon J, Sakzewski L. Hand function and self-care in children with cerebral palsy. Dev Med Child Neurol. 2021:63(5):576-83.

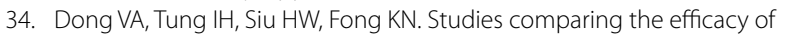
constraint-induced movement therapy and bimanual training in children with unilateral cerebral palsy: a systematic review. Dev Neurorehabil. 2013;16(2):133-43.

35. Facchin P, Rosa-Rizzotto M, Visonà Dalla Pozza L, Turconi AC, Pagliano E, Signorini S, et al. Multisite trial comparing the efficacy of constraintinduced movement therapy with that of bimanual intensive training in children with hemiplegic cerebral palsy: postintervention results. Am J Phys Med Rehabil. 2011;90(7):539-53.

36. Elvrum AG, Zethræus BM, VikT, Krumlinde-Sundholm L. Development and validation of the both hands assessment for children with bilateral cerebral palsy. Phys Occup Ther Pediatr. 2018;38(2):113-26.

\section{Publisher's Note}

Springer Nature remains neutral with regard to jurisdictional claims in published maps and institutional affiliations. 